\title{
Human colostrum oligosaccharides modulate major immunologic pathways of immature human intestine
}

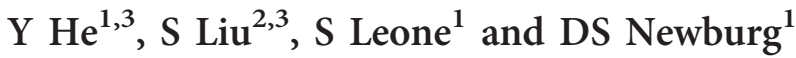

The immature neonatal intestinal immune system hyperreacts to newly colonizing unfamiliar bacteria. The hypothesis that human milk oligosaccharides from colostrum (cHMOSs) can directly modulate the signaling pathways of the immature mucosa was tested. Modulation of cytokine immune signaling by HMOSs was measured ex vivo in intact immature (fetal) human intestinal mucosa. From the genes whose transcription was modulated by cHMOSs, Ingenuity Pathway Analysis identified networks controlling immune cell communication, intestinal mucosal immune system differentiation, and homeostasis. cHMOSs attenuate pathogen-associated molecular pattern-stimulated acute phase inflammatory cytokine protein levels (interleukin-8 (IL-8), IL-6, monocyte chemoattractant protein-1/2 and IL-1ß), while elevating cytokines involved in tissue repair and homeostasis. In all, 3'-, 4-, and 6'-galactosyllactoses of cHMOSs account for specific immunomodulation of polyinosinic:polycytodylic acid-induced IL-8 levels. cHMOSs attenuate mucosal responses to surface inflammatory stimuli during early development, while enhancing signals that support maturation of the intestinal mucosal immune system.

\section{INTRODUCTION}

The intestinal mucosa defends against both pathogen infection (exogenous novel epitopes) and, conversely, autoimmunity (endogenous epitopes), in part by discriminating "self" from "non-self" antigens. ${ }^{1,2}$ The antigenic composition of the intestinal contents shifts rapidly after birth with the introduction of a plethora of novel antigens as dietary components, and as a consequence of early colonization by strains and species of microbiota novel to the newborn gut. ${ }^{2}$ This coincides with a period during which the immature neonatal intestinal mucosal immune system is hyperinflammatory. ${ }^{3}$ Subsequent immune maturation is required both for active responses to foreign antigens and for proper control and regulation of such responses. Impaired immunological development due to preterm delivery, injury at the mucosal surface, pathogenic infection, and food allergy may disturb the ontogeny of homeostatic control of inflammatory processes, contributing to conditions such as necrotizing enterocolitis (NEC), ${ }^{4,5}$ pediatric inflammatory bowel diseases, ${ }^{6}$ T helper cell 2 (Th2) disorders, and atopic dermatitis. ${ }^{6}$

Fortunately, human milk quenches inflammatory processes $^{7,8}$ and minimizes the incidence of many immune-based disorders. ${ }^{9}$ In early postnatal developing gut, milk enhances the signals that facilitate appropriate immune responses and antigenic memory. ${ }^{9}$ Moreover, human milk contains immune modulatory components and protects against development of inflammatory bowel diseases. ${ }^{10-12}$ Human milk oligosaccharides (HMOSs) are complex glycans containing a lactose moiety at the reducing end. At $5-15 \mathrm{mg} \mathrm{ml}^{-1}$, HMOSs are collectively the third most abundant solid component of human milk. ${ }^{13,14}$ Traditionally, up to 200 known individual HMOSs are subdivided into neutral and acidic oligosaccharides. ${ }^{13,14}$ Some possess anti-inflammatory functions, including reduced leukocyte adhesion, ${ }^{15}$ and some bind specifically to dendritic cells through the lectin receptor DC-SIGN (dendritic cell-specific intercellular adhesion molecule-3-grabbing non-integrin). ${ }^{16}$

\footnotetext{
${ }^{1}$ Program in Glycobiology, Department of Biology, Boston College, Chestnut Hill, Massachusetts, USA and ${ }^{2}$ Laboratory of Gynecologic Oncology, Department of Obstetrics and Gynecology, Brigham and Women's Hospital, Harvard Medical School, Boston, Massachusetts, USA. Correspondence: DS Newburg (david.newburg@bc.edu)

${ }^{3}$ These authors contributed equally to this work.
} 
The HMOS disialyllacto- $N$-tetraose reduces NEC-like inflammation in neonatal rats. ${ }^{17}$ Some HMOSs in colostrum, cHMOSs, are present at higher concentration and have a different pattern than HMOSs of mature milk (mHMOSs). ${ }^{18,19}$ Some oligosaccharides observed in colostrum decline to undetectable levels as lactation proceeds. ${ }^{20}$ The biologic activities of these colostrum-specific oligosaccharides has not been defined. Immature human intestine ex vivo was deemed the most relevant model for investigating effects of colostrum oligosaccharides on maturation- and inflammation-related signaling of the immature intestinal mucosa of neonates.

The hypothesis tested was that HMOSs from colostrum directly modulate mucosal signaling in immature human intestine. This was investigated in human fetal intestine explants. Changes induced by cHMOSs were determined in basal and pathogen-associated molecular pattern (PAMP) or interleukin-1 $\beta$ (IL-1 $\beta$ )-stimulated intestinal mucosa. The predominant Toll-like receptors (TLRs) expressed in intestinal mucosa are TLR3 and TLR5, ${ }^{21}$ whose PAMPs are doublestranded RNA (polyinosinic:polycytodylic acid, PIC) and flagellin (FLA), respectively. Stimulation of the IL- $1 \beta$ receptor mediates a variety of signaling pathways associated with innate immunity and host responses to microbes. Signaling was measured through reverse transcription-PCR array analysis of the cytokine-related transcriptome, and shifts in functional patterns of expression were confirmed by antibody arrays of the corresponding proteome, and enzyme-linked immunosorbent assay (ELISA) of IL-8 levels, a prototypic inflammatory cytokine of human intestinal mucosa. Immunomodulation by individual HMOS candidates was determined in cultured human intestinal epithelial cells.

\section{RESULTS}

Modulation of inflammatory cytokines and receptors in basal epithelium by cHMOSs

The initial condition of cultured fetal intestinal tissues was evaluated by IL- 8 concentrations in the media. As shown in Supplementary Figure 1 online, IL-8 concentrations were below $100 \pm 10 \mathrm{pg} \mathrm{ml}^{-1}$ in successfully cultured healthy tissue samples under quiescent conditions. This concentration represented basal expression of IL-8. When these intestinal samples were challenged with PAMPs (FLA and PIC) or the proinflammatory mediator IL- $1 \beta$, secretion of IL-8 increased (Supplementary Figure 1). Only intestinal samples with low basal IL-8 expression retain the ability to respond to exogenous proinflammatory stimuli, and these quiescent intestinal tissues were used in these experiments.

cHMOSs, but not mHMOSs $(P=0.036)$, directly modulated immunological signaling in the immature intestine, as measured using an 84-gene $\mathrm{RT}^{2} \mathrm{PCR}$ array with probes against chemokine and chemokine receptor genes. The screening criteria described in the Methods section identified 45 genes whose transcription was upregulated by cHMOSs and 11 genes whose transcription was downregulated by cHMOSs (Table 1). The 10 genes whose transcription was most upregulated and downregulated by the presence of cHMOSs are listed in
Table 1 Summary of HMOS-regulated inflammatory genes in human fetal intestine

\begin{tabular}{|c|c|c|c|c|c|}
\hline \multicolumn{2}{|c|}{ Chemokines and receptors } & \multicolumn{2}{|c|}{$\begin{array}{l}\text { Cytokines and } \\
\text { receptors }\end{array}$} & \multicolumn{2}{|c|}{$\begin{array}{l}\text { Other genes } \\
\text { involved in inflam- } \\
\text { matory responses }\end{array}$} \\
\hline Symbol & $\begin{array}{l}\text { Fold } \\
\text { change }\end{array}$ & Symbol & $\begin{array}{l}\text { Fold } \\
\text { change }\end{array}$ & Symbol & $\begin{array}{l}\text { Fold } \\
\text { change }\end{array}$ \\
\hline CCL2 (MCP-1) & 8.39 & IL1A & 13.4 & CARD18 & 35.46 \\
\hline CCL3 (MIP-1 $\alpha)$ & 4.24 & IL1B & 4.71 & CRP & 17.52 \\
\hline CCL11(eotaxin) & 72.74 & IL8 & 12.03 & $\mathrm{MIF}$ & 13.19 \\
\hline CCL13 & 16.62 & IL10 & 29.47 & LTB & 10.23 \\
\hline CCL16 & 5.20 & IL13 & 13.47 & $\mathrm{C} 4 \mathrm{~A}$ & 6.11 \\
\hline CCL20 (MIP-3 $\alpha)$ & 5.28 & IL17C & 9.97 & IFNA2 & 4.77 \\
\hline CCL21 (MIP-2) & 208.61 & IL36G & 4.75 & AlMP1 & 3.96 \\
\hline CCL23 & 3.81 & IL37 & 14.40 & CEBPB & 3.71 \\
\hline $\begin{array}{l}\text { CCL24 (eotaxin- } \\
\text { 2) }\end{array}$ & 41.97 & IL1R1 & 5.09 & BCL6 & 0.02 \\
\hline CCL25 & 6.56 & IL1RN & 3.98 & & \\
\hline CXCL2 & 33.31 & IL5RA & 4.93 & & \\
\hline CXCL3 & 3.8 & IL10RA & 12.1 & & \\
\hline CXCL6 & 8.04 & IL10RB & 15.61 & & \\
\hline CXCL9 & 5.07 & IL13RA1 & 6.73 & & \\
\hline CXCL10 (IP-10) & 21.58 & IL36RN & 4.17 & & \\
\hline CXCL11 & 13.13 & IL5 & 0.22 & & \\
\hline CXCL13 (MCP-4) & 13.5 & IL9R & 0.34 & & \\
\hline CXCL14 & 7.4 & & & & \\
\hline CCR4 & 3.56 & & & & \\
\hline CCR6 & 16.46 & & & & \\
\hline CCR8 & 4.22 & & & & \\
\hline CX3CR1 & 6.52 & & & & \\
\hline CCL1 (I-309) & 0.38 & & & & \\
\hline CCL4 (MIP-1 $\beta)$ & 0.78 & & & & \\
\hline 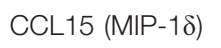 & 0.57 & & & & \\
\hline CCL17 & 0.66 & & & & \\
\hline CCL19 & 0.43 & & & & \\
\hline CCR1 & 0.37 & & & & \\
\hline CCR2 & 0.77 & & & & \\
\hline CXCR2 & 0.58 & & & & \\
\hline
\end{tabular}

Abbreviations: AIMP, aminoacyl tRNA synthetase complex-interacting multifunctional protein; BCL6, B-cell CLL/lymphoma 6; CCL, chemokine (C-C motif) ligand; CEBPB, CCAAT/enhancer binding protein; CRP, C-reactive protein; CXCL, chemokine (C-X-C motif); CCR, chemokine (C-C motif) receptor: CX3CR, chemokine (C-X3-C motif) receptor; C4A, complement component 4A; HMOS, human milk oligosaccharide; IFN, interferon; IL, interleukin; MIF, macrophage migration inhibitory factor; LTB, lymphotoxin beta; RM ANOVA, repeated-measures analysis of variance. Data represent the fold changes ( $>3.5$ or $<0.8 ; P<0.05$ ) of messenger RNA expression of cytokine, inflammatory regulator, cytokine receptor, chemokine, and chemokine receptor genes in human fetal intestine in response to 2 days of HMOS exposure. Significance of differences $(P)$ was measured by two-way RM ANOVA (Graph Pad Software).

Figure 1. Those genes whose transcription was highly upregulated include several chemokines, such as CCL21, CCL11, CCL24, CXCL2, CXCL10, and CCL13. IL-10, usually 


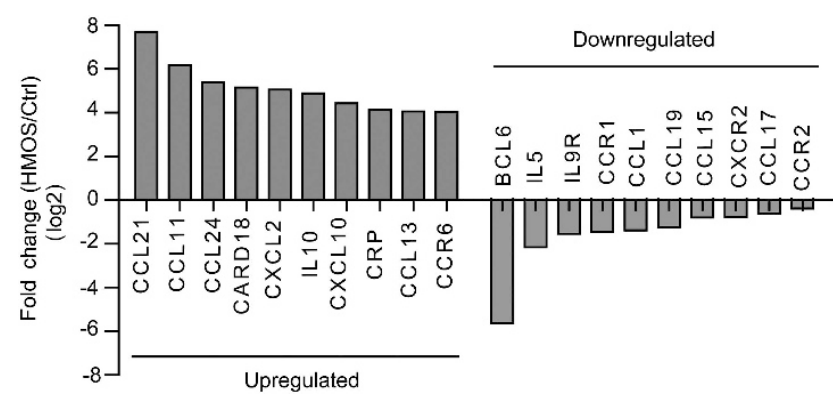

Figure 1 Genes whose transcription is most modulated by human milk oligosaccharides from colostrum (cHMOSs). Red bars, 10 genes whose transcription is most enhanced by cHMOSs; blue bars, 10 genes whose transcription is most attenuated by cHMOSs. A full color version of this figure is available at the Mucosal Immunology journal online.

considered an anti-inflammatory cytokine, also increased. Another significantly upregulated gene transcription was CARD18 (caspase recruitment domain-containing protein, also called caspase- 1 inhibitor Iceberg), which inhibits generation of IL-1 $\beta$ through interaction with caspase- 1 and prevents it from forming a complex with receptor interacting protein $2 .^{22}$ Among the genes whose transcription was downregulated by cHMOSs, BCL6 and IL-5 were the most highly suppressed, followed by chemokine receptors CCR1, CXCR2, and CCR2, and chemokines CCL1, CCL19, CCL15, and CCL17.

\section{Analysis of cHMOS-modulated gene expression in basal intestinal mucosa}

cHMOS-induced changes in gene transcription were clustered computationally by Ingenuity Pathway Analysis (IPA) according to known canonical pathways and biofunctional networks. This program analyzes functional relationships among cHMOS-modulated genes within known signaling pathways. The discrete canonical pathways most strongly modified by cHMOSs (Figure 2) include those for T-helper (Th) cell differentiation, immune cell communication, regulation of innate immunity, communication between innate and adaptive immune cells, and dendritic cell maturation.

IPA also identified four higher order functional networks composed of specific biological pathways of cytokine and chemokine genes whose transcription was strongly modified by cHMOSs (Figure 3). A pathway involved in immune cell trafficking is depicted in Figure 3a, in which extracellular signal-regulated kinases $1 / 2$ (ERK1/2) is the central signaling molecule. This network includes several genes whose transcription is strongly influenced by cHMOSs, including upregulation of CCL21, CCL11, and CRP, and attenuation of CCL19 expression. A network modulating development and function of specific cells of the hematological system, as well as antigen presentation, is shown in Figure $\mathbf{3 b}$. In this network, the genes whose transcription is stimulated by cHMOSs include CCL24, CXCL12, CCR6, and those whose transcription is attenuated by cHMOSs include CCR1, CXCR2, CCL1, and CCL17. For these two networks, cHMOS treatment is expected to have specific effects on various constituent pathways.

A network that regulates inflammatory response is illustrated in Figure 3c, in which cHMOSs stimulate expression of CXCL2, CXCL10, and IL-10. Calculated global cytokine change scores in this network deduce a potential increase in Th1 cytokines. The genes upregulated by cHMOSs, which lead to a predominance of cytokines supporting a Th1 response, are labeled red.

In the network of genes that control signaling pathways for development and function of specific cell types during hematological system development (Figure 3d), cHMOSs attenuated transcription of $B C L 6, I L-5$, and $I L 9 R$. The deduced cytokine expression changes suggest a global change of cytokines that would attenuate Th2 cytokine functions. Genes whose expression is attenuated by cHMOSs, and whose decrease would suppress cytokines supporting Th2 function, are labeled green.

\section{cHMOS-modulated cytokine expression in basal immature intestinal mucosa}

The above analyses of the functional significance of cHMOS modulation of signaling systems in quiescent basal mucosa were deduced from differences in gene transcription. Changes in expression of inflammatory cytokine protein levels induced by cHMOSs in immature intestine were measured to scrutinize the conclusions that had been based on RNA levels. The cytokines were measured using antibody membrane arrays that detect 40 different cytokines simultaneously. In Supplementary Figure 2, the position map of cytokines and the corresponding intensity of binding are shown. The change in expression of signaling molecules induced by cHMOSs in basal unstimulated intestinal mucosa is summarized in Figure 4. cHMOSs suppressed translation of IL-17, IL-13, IL-4, IL-2, IL-11, granulocyte colony-stimulating factor, tumor necrosis factor (TNF)- $\beta$, transforming growth factor- $\beta 1$, granulocyte-macrophage colony-stimulating factor, and eotaxin. Among these are typical Th2-polarizing cytokines IL-13, IL-4, IL-1 $\beta$, and IL-6. Similarly, the expression of Th17-polarizing cytokines IL-17, TNF- $\beta$, transforming growth factor- $\beta 1$, and IL- 4 was reduced by cHMOSs. In contrast, translation of Th1-polarizing cytokines monocyte chemoattractant protein (MCP)-1 (CCL-2) and IL12-p70 was stimulated by cHMOSs. The overall functional pattern of cHMOS modulation of signaling molecule translation is consistent with the overall functional pattern of the changes in transcription observed in the reverse transcription-PCR arrays. The concordance between the increase in MCP-1 protein and upregulation of its mRNA (CCL2) transcription is striking. However, decreased cytokine protein levels, such as IL-10, did not vary according to changes in their mRNA transcription following cHMOS treatment; this suggests that these cytokine protein levels may be regulated by factors other than gene transcription, such as the presence of miRNA, rates of degradation, or posttranslational modifications, which are common in regulation of the rapidly changing levels of signal transduction molecules. ${ }^{23}$ That notwithstanding, the imputed 


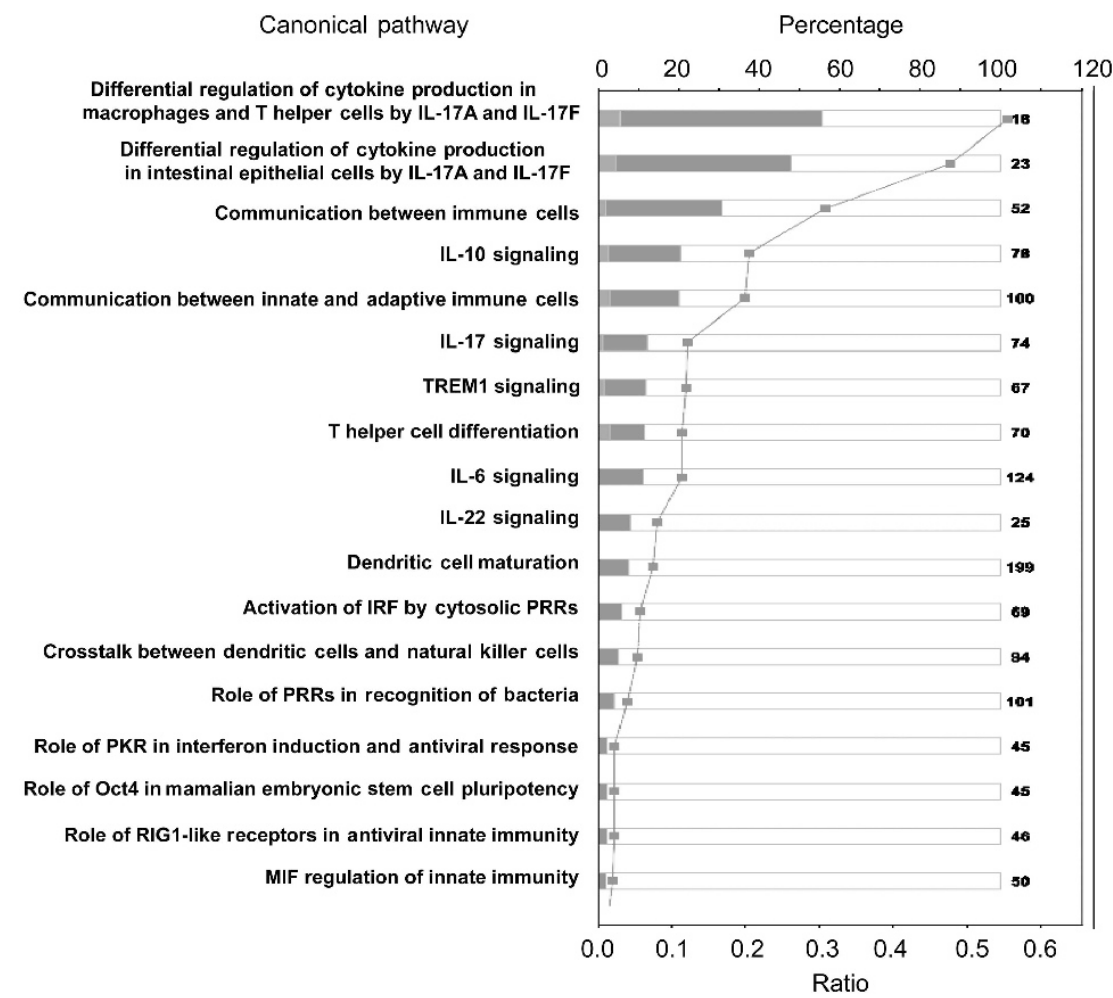

Figure 2 Canonical pathways whose genes are most strongly modulated by human milk oligosaccharides from colostrum (cHMOSs). Canonical pathways whose transcription is influenced by cHMOS treatment were identified by Ingenuity Pathway Analysis (IPA) and rank ordered by the magnitude of their ratios. The names of canonical pathways are presented on the y axis. Red bars represent genes whose transcription is enhanced by cHMOS treatment; green bars, genes whose transcription is attenuated by cHMOSs. Orange squares represent ratios calculated from changes in gene expression from Table 1. A full color version of this figure is available at the Mucosal Immunology journal online.

global functional consequences of the protein and mRNA changes in response to cHMOSs are congruent. Both suggest that cHMOSs modulate differentiation of Th cells toward a balanced Th1 expansion in unstimulated basal immature intestine.

\section{cHMOS-modulated cytokine expression after proinflammatory stimulation of immature intestinal mucosa}

To query whether $\mathrm{cHMOS}$ influence innate immune responses in inflamed immature intestine, the tissues were treated with three distinct PAMP-related inflammatory stimuli, FLA, PIC, and IL- $1 \beta$, at concentrations previously found to be effective inducers of inflammatory signaling in this system. ${ }^{24}$ Figure 5 illustrates that cHMOSs significantly modulated a wide spectrum of cytokine protein expression induced by PAMP or IL-1 $\beta$ stimulation. Several cytokines were upregulated by cHMOSs. In tissues challenged with FLA, cHMOSs

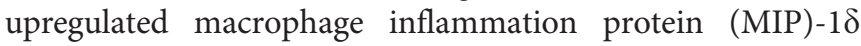
(CCL15) and intercellular adhesion molecule-1 expression (Figure 5a). Each of these promotes the uptake of bacterial pathogens by recruiting macrophages and neutrophils, and lead to bacteria clearance. ${ }^{25,26}$ In intestine challenged with PIC, cHMOSs upregulated tissue inhibitor of matalloproteinase-2 and platelet derived growth factor-BB (Figure 5c), both of which enhance the regulation of epithelial cell functions and promote mucosal repair. ${ }^{27}$ In intestine stimulated by IL-1 $\beta$, cHMOSs upregulated expression of MIP- $1 \delta$ (CCL15), MIP-1 $\beta$ (CCL4), and tissue inhibitor of matalloproteinase-2 (Figure 5b). Tissue inhibitor of matalloproteinase-2 inhibits matrix metalloproteinase, and is critical in maintaining tissue homeostasis. ${ }^{28}$

Although modulation of cytokine expression by cHMOSs varied in tissues stimulated by different PAMP-related stimuli, those intracellular cytokines mediating acute phase inflammation, including IL- 8 , IL- 6 , IL-1 $\beta$, TNF $(-\alpha$ or $-\beta)$, and MCP $(-1$ or -2$)$, were suppressed by cHMOSs in all cases (Figure 5a-c). This ability of cHMOSs to attenuate PAMPinduced inflammation was corroborated by measuring IL-8 secretion by ELISA in the tissue supernatant. IL- 8 is a chemokine produced by macrophages and epithelial cells of the intestinal mucosa. As a prototypic marker of inflammation, IL-8 recruits neutrophils to sites of inflammation in response to infection, trauma, ischemia, and cancer. ${ }^{29}$ The basal IL-8 concentration is not significantly changed by cHMOSs. PAMPrelated stimuli increase IL-8 secretion in immature intestine. These increased IL- 8 secretions were abrogated by the presence of cHMOSs (Figure 5d). In FLA $\left(100 \mathrm{ng} \mathrm{m}^{-1}\right)$ stimulated tissue, cHMOS treatment significantly reduced IL-8 expression by approximately $25 \%(P<0.05)$. In PIC $\left(10 \mathrm{ng} \mathrm{ml}^{-1}\right)$ and IL- $1 \beta$ $\left(10 \mathrm{ng} \mathrm{ml}^{-1}\right)$ stimulated tissues, cHMOSs suppressed IL-8 expression markedly, from concentrations of $235 \mathrm{pg} \mathrm{ml}^{-1}(P<0.05)$ and 
a

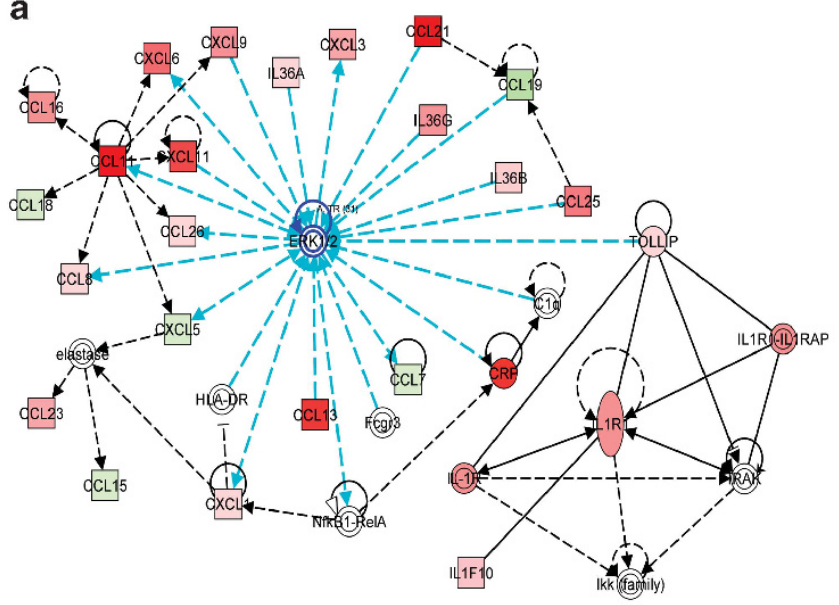

C

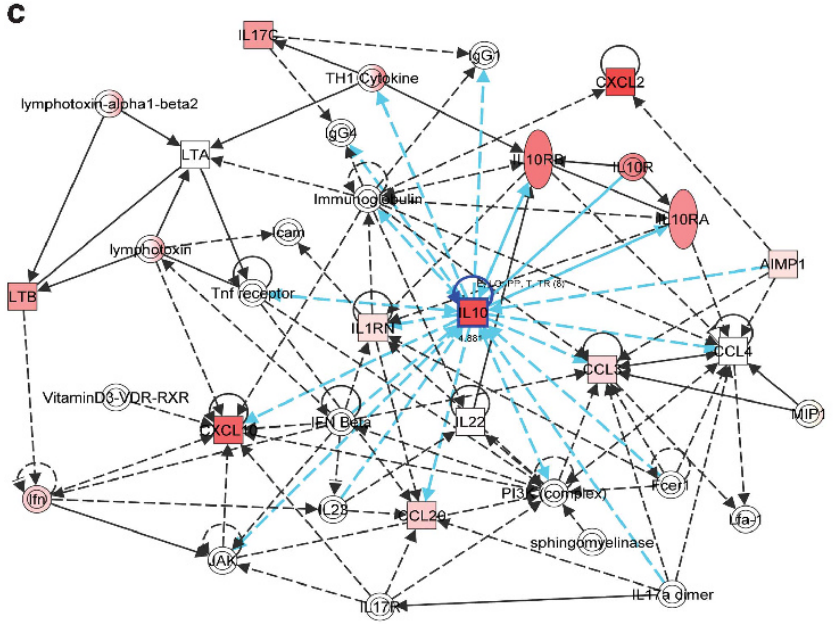

b
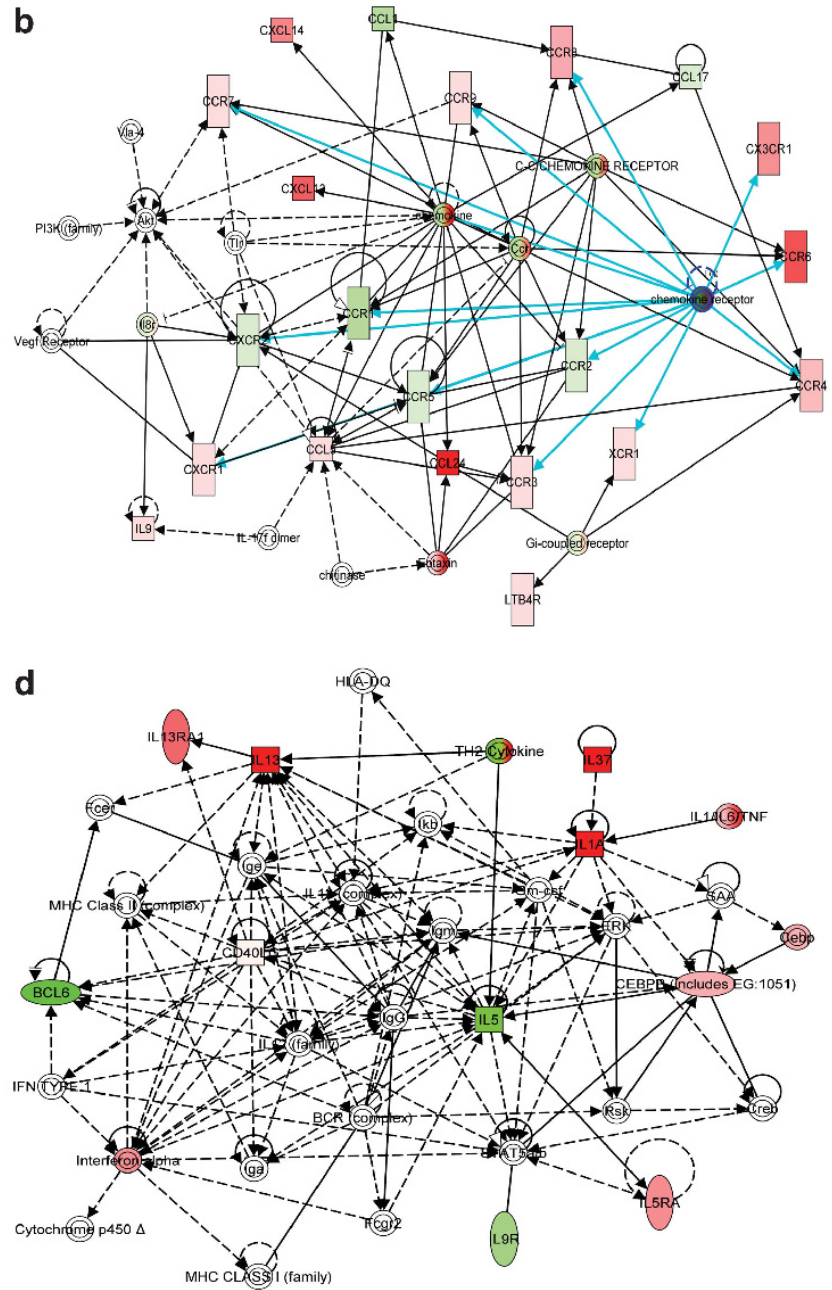

Figure 3 Four functional networks whose gene expression was most modulated by human milk oligosaccharides from colostrum (cHMOSs). (a) Network 1 signaling pathways for "cell/cell interaction, cellular movement, and immune cell trafficking". (b) Expression products of the genes of Network 2 comprise signaling pathways for "antigen presentation, hematological system development and function". (c) Network 3 genes for cytokines promoting T helper cell 1 (Th1) activity and differentiation that were stimulated by cHMOSs. (d) Network 4 genes for cytokines promoting Th2 activity and differentiation were suppressed by cHMOSs. Solid lines, direct interaction; dashed lines, indirect interaction. Red indicates gene transcription promoted by cHMOSs; green indicates gene transcription suppressed by cHMOSs.

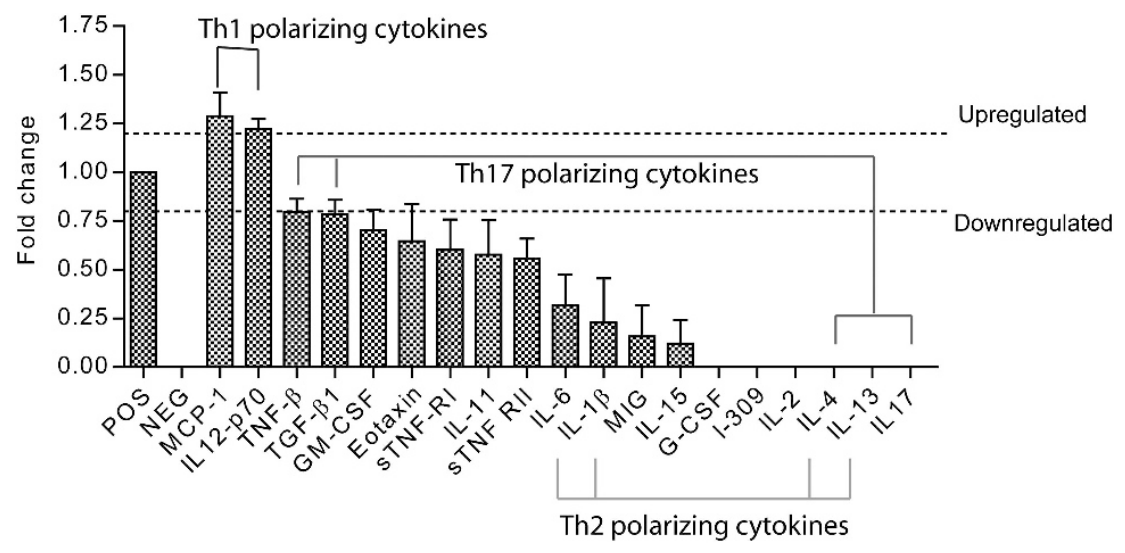

Figure 4 Modulation of cytokine translation by human milk oligosaccharides from colostrum (cHMOSs) in quiescent unstimulated mucosa. Cytokine translation was assessed by antibody membrane array. Cytokine expression in quiescent mucosa was significantly modulated by cHMOSs, with changes of $>1.2$-fold considered upregulated and changes of $<0.8$ considered downregulated (comparison with basal levels, $P<0.05$ ). 

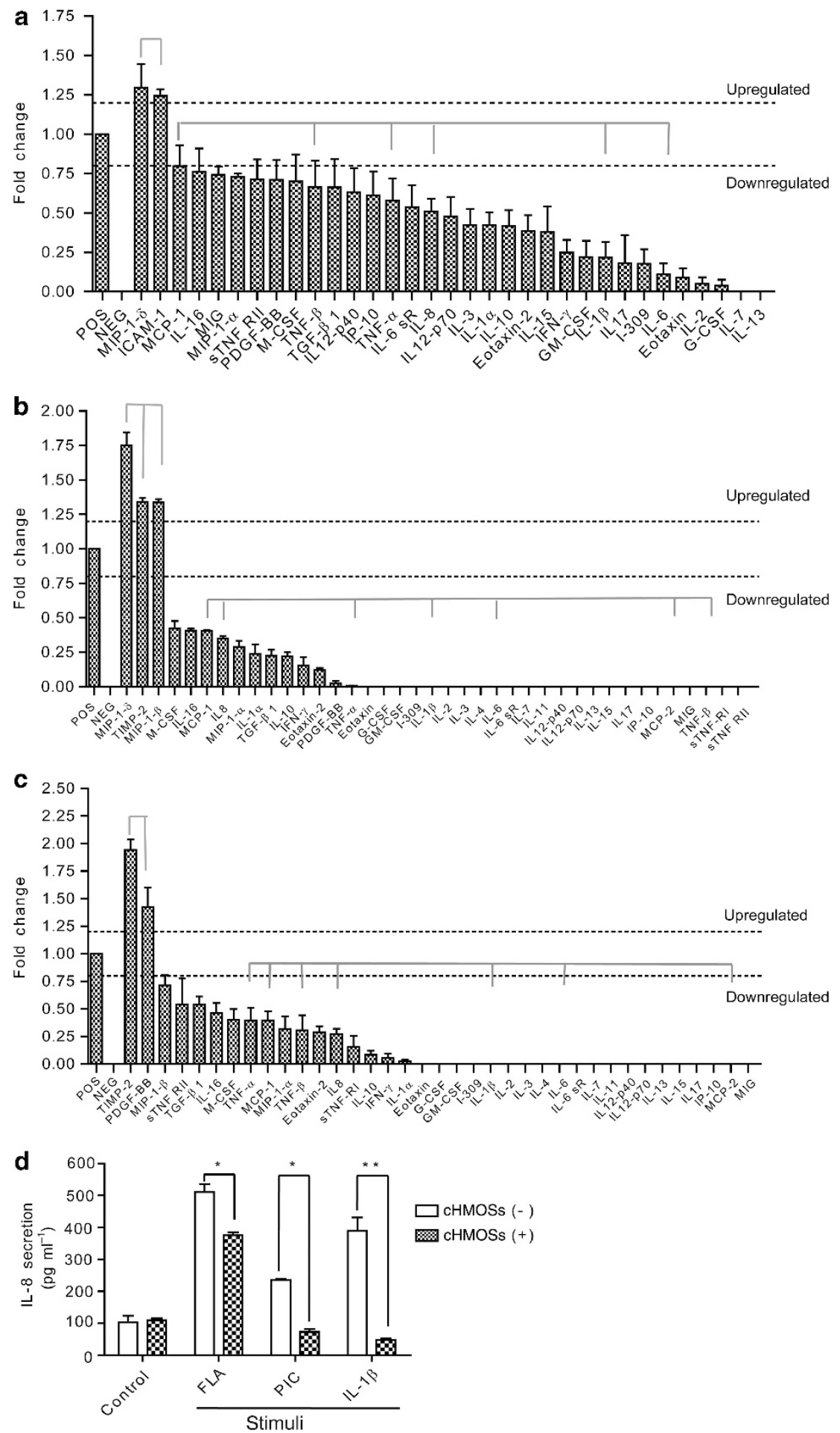

Figure 5 Modulation of cytokine translation by human milk oligosaccharides from colostrum (cHMOSs) in pathogen-associated molecular pattern (PAMP)-stimulated mucosa. (a) cHMOSs modulated the flagellin (FLA)-induced changes in cytokine profile of immature intestinal mucosa. (b) cHMOSs modulated the IL-1 $\beta$-induced changes in cytokine profile of immature intestinal mucosa. (c) cHMOSs modulated the polyinosinic:polycytodylic acid (PIC)induced changes in cytokine profile of immature intestinal mucosa. Green lines indicate acute inflammatory cytokines suppressed by cHMOSs. Red lines indicate gene translation promoted by cHMOSs. Data were duplicates from three independent experiments $(n=6, P<0.05)$. (d) cHMOS treatment attenuated the stimulation of interleukin-8 (IL-8) cytokine secretion induced by these three major PAMP-related proinflammatory molecules. $\left({ }^{\star} P<0.05\right.$; $\left.{ }^{\star \star} P<0.01\right)$. A full color version of this figure is available at the Mucosal Immunology journal online.

$384 \mathrm{pg} \mathrm{ml}^{-1}(P<0.01)$, respectively, to basal levels of expression $\left(100 \mathrm{pg} \mathrm{ml}^{-1}\right)$. Attenuation of secreted IL-8 measured by ELISA was consistent with the results of the cytokine membrane array, demonstrating that the cHMOS fraction is anti-inflammatory, protecting immature infant intestinal mucosa from inflammation triggered by various PAMP or PAMP-related stimuli. 


\section{LC-MS profile of active HMOSs}

The quantities and concentrations of individual oligosaccharides vary between individuals and over the course of lactation. ${ }^{14,19}$ HMOSs of different mothers can vary in activity. ${ }^{19}$ In preliminary experiments, different milk preparations varied greatly in their ability to quench inflammation (manifested as elevated IL-8 levels). Therefore, the differences between early milk and mature milk oligosaccharides were studied systematically as part of the experimental paradigm. Of the eight HMOS preparations in this study, only the two that had been prepared from colostrum (cHMOSs) demonstrated the robust and consistent immune modulatory activities in fetal intestinal tissue shown above $(P=0.036)$. The HMOS preparations, six from mature milks and two from colostrum, were each prepared from milks pooled from a discrete population of 20-50 mothers. The individual oligosaccharides of each were resolved and quantified by LC-MS (high-performance liquid chromatography with tandem mass spectrometry). All eight preparations contained the typical pattern of HMOSs of pooled milk, including a preponderance of $2^{\prime}$-fucosyllactose, lacto- $N$ fucopentaose I, lacto- $N$-tetraose, and lactodifucotetraose (Figure 6a). However, the two cHMOS preparations contained higher concentrations of several larger oligosaccharides whose structures terminate with complex fucosylated and/or sialylated moieties, as well as two distinct peaks of galactosyloligosaccharides previously reported only in colostrum, $6^{\prime}$ - and $3^{\prime}$-galactosyllactoses ${ }^{20}$ (Figure 6a). The variation in amounts of individual oligosaccharides among the eight preparations is shown in Figure $\mathbf{6 b}$.

The higher concentration of galactosyllactoses in cHMOSs relative to mHMOSs, when considered together with immunomodulation by only cHMOSs, suggested galactosyllactoses as candidate molecules for immunomodulation. The $3^{\prime}$ - and $6^{\prime}$ galactosyllactoses that were detected in the active cHMOS fractions, as well as the 4-galactosyllactose reported to be in human colostrum, were tested at a concentration of $200 \mu \mathrm{g} \mathrm{ml}^{-1}$ for immunomodulation and compared with cHMOSs at $5 \mathrm{mg} \mathrm{ml}^{-1}$. These concentrations are within the physiologic range, given the highly variable expression of HMOSs in colostrum. The activities were tested in $\mathrm{H} 4$ cells, a line of immature intestinal epithelial cells that provide an expedient and relevant model for screening these activities, as $80 \%$ of the cells in the 18- to 22-week-old intestinal mucosa are intestinal epithelial cells. ${ }^{30}$ Exposure of the epithelial cells to proinflammatory PAMPs (FLA or PIC) or IL-1 $\beta$ at levels previously found to be effective in cell culture (unpublished data) induced pronounced increases in IL-8 levels. None of the three galactosyllactoses attenuated the IL- 8 inflammatory responses to FLA or IL-1 $\beta$ (Figure $6 \mathbf{c}$ and $\mathbf{d}$ ). In contrast, the IL- 8 response to $2 \mu \mathrm{g} \mathrm{ml}^{-1}$ PIC was significantly attenuated specifically by $3^{\prime}$-galactosyllactose, but not 4 - or 6 '-galactosyllactoses relative to the phosphate-buffered saline (PBS) control group (Figure 6e). However, if the cells stimulated by $2 \mu \mathrm{g} \mathrm{ml}^{-1}$ PIC and treated with mHMOSs (which exhibited no inhibition) are combined with the PBS-treated positive control cells also stimulated by $2 \mu \mathrm{g} \mathrm{ml}^{-1} \mathrm{PIC}$, then relative to this expanded control group, $3^{\prime}$-, 4-, and $6^{\prime}$-galactosyllactose inhibition was significant at $P=0.001,0.03$, and 0.03 , respectively. When the stimulation was reduced to $200 \mathrm{ng} \mathrm{ml}^{-1}$ PIC (Figure 6f), the induction of IL- 8 was inhibited by each of the three galactosyloligosaccharides at 75,100 , and $200 \mu \mathrm{g} \mathrm{ml}^{-1}$. Thus, galactosyllactose replicates the ability of the native cHMOS fraction to attenuate the IL-8 inflammatory response to PIC at physiologically meaningful concentrations in vitro, but the remaining activities of the cHMOS fraction cannot be accounted for by the galactosyllactoses tested, indicating that additional oligosaccharide components of cHMOS remain candidates for mediating the residual immunomodulation exhibited by cHMOSs.

\section{DISCUSSION}

The intestine is the largest immune organ in the body and is proximate to a diverse pool of immune effector cells. In comparison with intestinal epithelial cells of the mature mucosa, neonatal intestinal epithelial cells overexpress innate inflammatory genes, such as $N F \kappa B, M y D 88, T L R 2, T L R 4$, and $T R A F$, with scant expression of negative feedback regulator genes. ${ }^{31}$ Accordingly, immature intestinal mucosa is prone to exaggerated responses to proinflammatory stimuli, increasing the risk of inflammatory diseases of the intestine in the neonate. ${ }^{31}$

Immature infant intestinal mucosa also contain fewer total $\mathrm{T}$ cells than mature mucosa, ${ }^{32}$ and those present are skewed toward a Th2 bias. During pregnancy, Th2 responses prevent adverse immunological reactions between the mother and her fetus $^{33}$ (Figure 7a). This residual Th2 bias leaves neonatal intestinal mucosa more vulnerable to bacterial infection and food allergy, ${ }^{34}$ and the intestinal immune system must mature to meet immune challenges of adult life.

The hypersensitivity to inflammatory stimuli and the skew toward Th2 are major contributors toward the extraordinary reactiveness of the neonatal, and especially premature, intestinal mucosa to external stimuli. Breastfeeding and human milk per se are known to counter adverse outcomes associated with inappropriately high responses to mucosal stimulation. For example, feeding human milk to preterm infants lowers incidence and severity of infectious and autoimmune diseases. $^{12,35}$ Many human milk components are biologically active, including anti-inflammatory cytokines, chemokines, hormones, and growth factors. ${ }^{36,37}$ The natural mixture of HMOSs alters cytokine production by $\mathrm{T}$ cells from cord blood. $^{38}$ Plant-derived oligosaccharides designed to mimic those of human milk increase the Th1 response of Th2-skewed populations. ${ }^{38}$ Thus, human milk components, especially HMOSs, are putative external agents that induce tolerance, protecting infants from allergy development, while supporting maturation of the infant's immune system. ${ }^{7,39,40}$ The studies herein directly tested the ability of cHMOSs to modulate signaling in human immature intestinal mucosa ex vivo and identified signaling pathways that provide potential mechanisms for such activity. 

a b Ratio of abundance (cHMOS/mHMOS)

Mass intensity (specific ion monitoring)

mHMOS $=1$

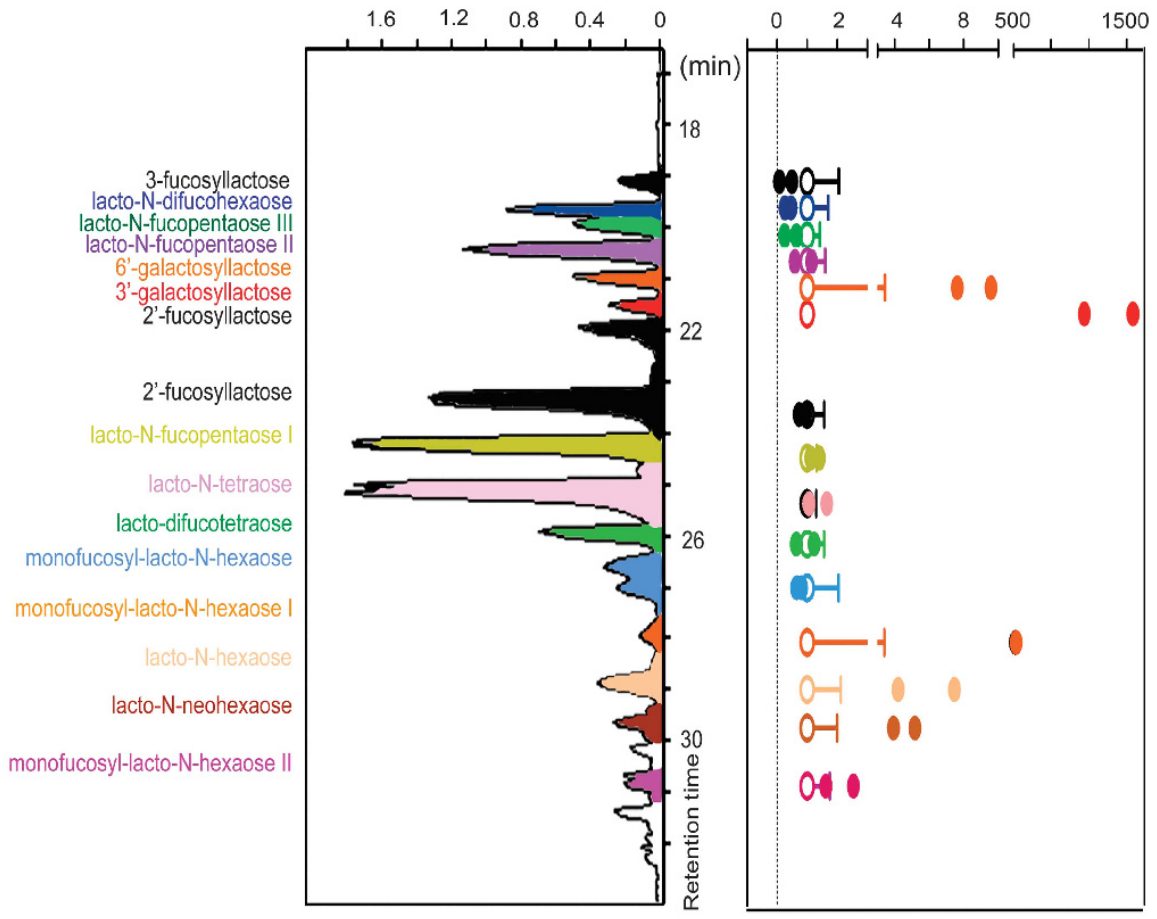

c

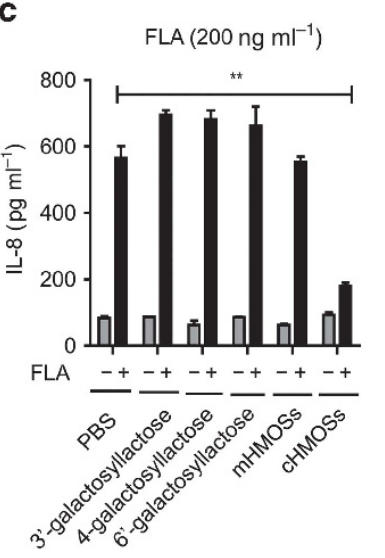

d

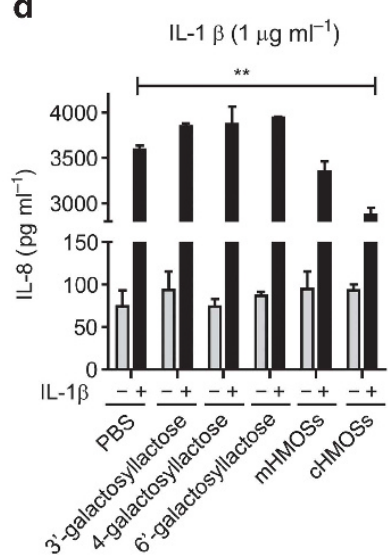

e

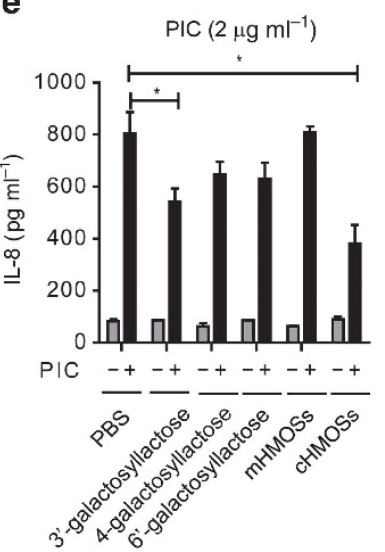

PIC (200 ng ml-1)

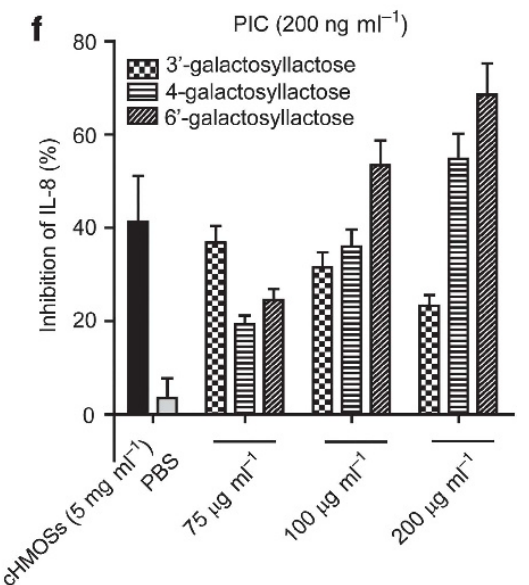

Figure 6 Characterization of active human milk oligosaccharide (HMOS) profile by LC-MS (high-performance liquid chromatography with tandem mass spectrometry). (a) Representative profile of HMOSs from colostrum by LC-MS. Major peaks were identified by comparing retention times and mass spectra with authentic isolated standards. (b) Variation in relative amounts of the major oligosaccharides isolated from mature human milk (open circles) and colostrum (filled circles). The two colostrum preparations were highly active immunomodulators, as described above, while the mature milk HMOSs did not display consistent robust activity. (c) Inhibition by galactosyllactoses relative to HMOSs from colostrum and mature milk on interleukin-8 (IL-8) secretion in flagellin (FLA; $200 \mathrm{ng} \mathrm{ml}^{-1}$ ) stimulated immature intestinal epithelial cells. (d) Inhibition by galactosyllactoses relative to HMOSs from colostrum and mature milk on IL-8 secretion in IL-1 $\beta\left(1 \mu \mathrm{g} \mathrm{ml}^{-1}\right)$ stimulated immature intestinal epithelial cells. (e) Inhibition by galactosyllactoses relative to HMOSs from colostrum and mature milk on IL-8 secretion in PIC $\left(2 \mu \mathrm{g} \mathrm{ml}^{-1}\right)$ stimulated immature intestinal epithelial cells. (f) Dose-related inhibition by galactosyllactoses $\left(75-200 \mathrm{~g} \mathrm{ml}^{-1}\right)$ relative to colostrum HMOSs (cHMOSs) on IL-8 secretion in PIC $\left(200 \mathrm{ng} \mathrm{ml}^{-1}\right)$ stimulated immature intestinal epithelial cells.

Development of lymphoid tissues, including differentiation of $\mathrm{T}$ lymphocytes, is a complex process regulated by diverse cytokine signals. ${ }^{41}$ In the ex vivo human immature intestinal mucosa, cHMOSs-modulated expression of a wide range of cytokines at both transcription (mRNA) and translation (protein) levels (Figures 1 and $\mathbf{4}$ and Table 1). To interpret these changes in the context of discrete immunological signaling pathways, they were clustered into known canonical pathways (recognized pathways from the literature) and biofunctional networks (imputed de novo from the data) by the IPA software system. This analysis of the data, discussed below, strongly suggests that in basal, unstimulated immature intestinal mucosa (a model for early life, especially for the extremely low birthweight neonate), cHMOSs modulate immune cell trafficking, maturation, and balance. 
a
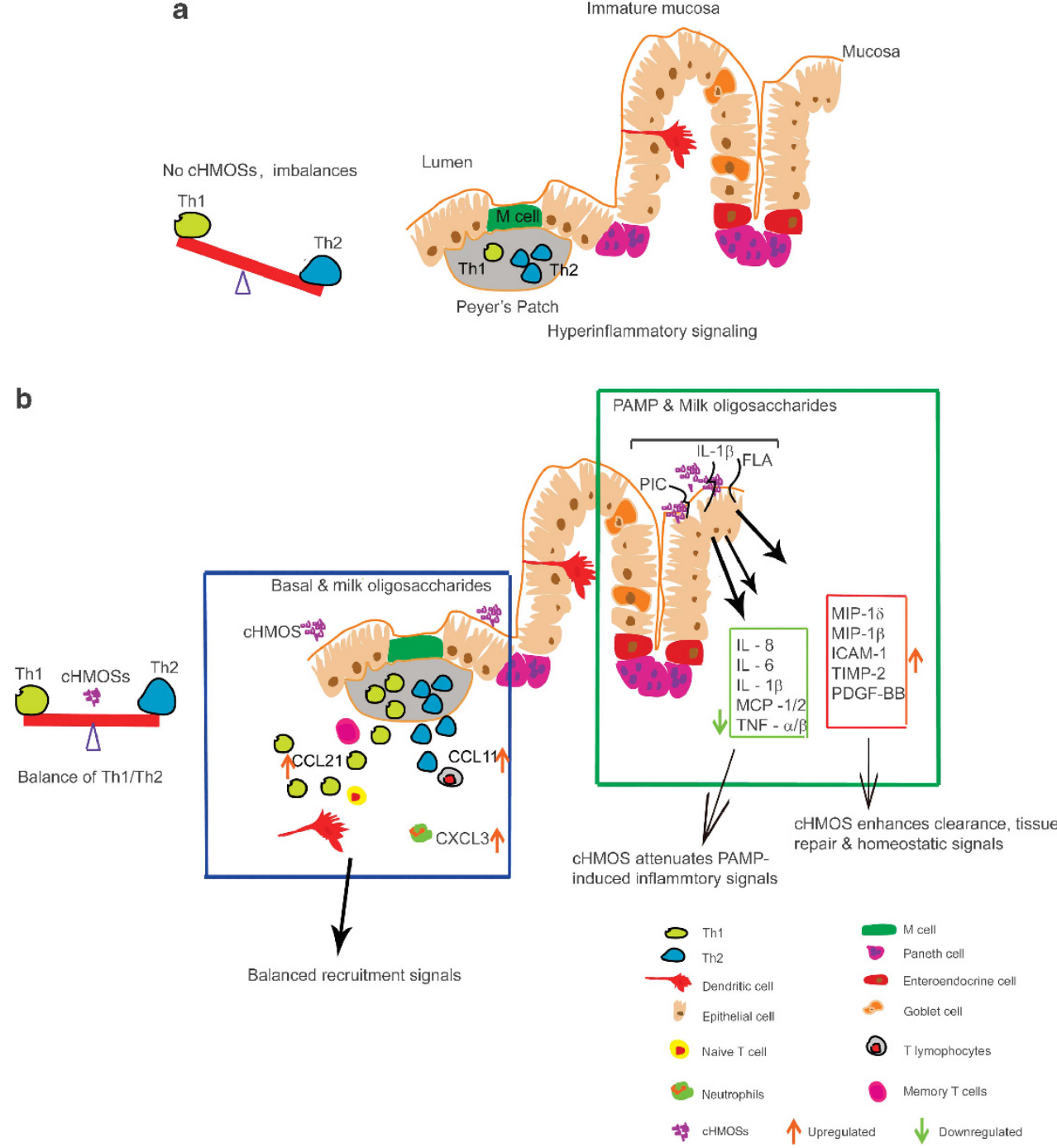

Figure 7 Hypothetical model of colostrum human milk oligosaccharide (cHMOSs) as immune modulators in human fetal intestine. (a) The neonatal intestinal immune system is immature at birth with an imbalance between Thelper cell 1 (Th1)-Th2 and fewer T cells. (b) Multiple effects of cHMOSs on immune modulation in immature intestine.

cHMOS modulate intrinsic expression of inflammatory markers associated with cell trafficking. In basal, unstimulated mucosa, cHMOSs enhanced expression of cytokines associated with recruitment of immune cells, including CCL21, CCL11, CCL13, and CXCL3 (Table 1). CCL21 recruits CCR7-bearing naive $\mathrm{T}$ cells, natural killer cells, memory $\mathrm{T}$ cells, and dendritic cells. ${ }^{42}$ CCL11 recruits lymphocytes bearing CCR3 ${ }^{43}$ CCL13 (MCP-4) participates in recruitment of monocytes, eosinophils, T lymphocytes, and basophils. ${ }^{44}$ CXCL3 (MIP-2 $\beta$ ) attracts neutrophils. ${ }^{45}$ The common downstream molecule of these genes is ERK1/2 (ERK, mitogen-activated protein kinase), whose ERK activity is central to signal pathways mediating immune cell trafficking ${ }^{46}$ (Figure 3c).

cHMOSs also modulate signaling pathways related to maturation of lymphoid tissue (Figure 2). Genes whose expression was significantly changed by cHMOSs included signaling molecules for key canonical pathways of immune maturation, including Th cell differentiation, dendritic cell maturation, and immune cell intercommunication, especially communication between innate and adaptive immune cells. As in the trafficking pathway above, again ERK1/2 is a signaling molecule identified by IPA analysis (Figure 3a) as one of the most strongly affected by cHMOSs. However, in this pathway its mitogen-activated protein kinase activity has a central role in promoting cellular proliferation. ${ }^{47}$ This suggests that cHMOSs stimulate ERK1/2 pathway signaling cytokines that promote intestinal immune system development (Figure $7 \mathbf{b}$ ) through rapid lymphoid tissue expansion. ${ }^{48}$

In this basal, unstimulated mucosal tissue, cHMOSs also influenced cytokine and chemokine networks that regulate Th1/Th2 lymphocyte balance (Figure $7 \mathbf{b}$ ). Th1 cells drive the type-1 pathway ("cellular immunity") to fight viruses and other intracellular pathogens and to expunge cancerous cells. ${ }^{49} \mathrm{Th} 2$ cells drive the type- 2 pathway ("humoral immunity") to 
upregulate antibody production to fight extracellular organisms. ${ }^{50}$ However, Th1 cells secrete cytokines that inhibit Th2 proliferation, while Th2 secrete cytokines that inhibit Th1 cell proliferation, ${ }^{50}$ a reciprocal inhibition that is essential for immune homeostasis. Among the cHMOS upregulated genes, CCL21 recruits CCR7-bearing Th1 cells, ${ }^{51}$ whereas IL-36 signaling enhances Th1 proliferation and Th1 polarization of naive CD $4+$ T cells. $^{52}$ Although cHMOSs upregulate CCL11, which induces recruitment of CCR3-bearing Th2 cells, ${ }^{43}$ it attenuates BCL- 6 and IL-5, regulators of differentiation of germinal center $\mathrm{B}$ cells, ${ }^{53,54}$ suppressing differentiation of $\mathrm{B}$ cells. Downstream, cHMOS treatment stimulates Th1 cytokine production, while suppressing Th2 cytokine production (Figure $3 \mathbf{c}$ and $\mathbf{d}$ ). These cHMOS-related changes in functional patterns of mRNA in quiescent mucosal tissues were confirmed by cytokine (protein) membrane array. Archetypal Th2 and Th17-polarizing cytokines are suppressed by cHMOSs, whereas Th1-polarizing cytokines are upregulated (Figure 4). The improved balance between Th1 and Th2 fostered by cHMOSs may enhance defense against pathogen infection and attenuate susceptibility to food allergy and autoimmune diseases in neonatal intestinal mucosa.

Profuse expression of innate inflammatory genes and sparse expression of negative feedback regulator genes ${ }^{31}$ underlies the bias toward production of proinflammatory cytokines, including IL- 6 and IL-8, by basal unstimulated immature mucosa. ${ }^{32}$ IL- 6 and IL- 8 are acute phase mediators of the innate immune response $\mathrm{e}^{33,55}$ in recruiting neutrophils. Neutrophils are the most abundant leukocyte in humans and kill microorganisms in an immediate, nonspecific manner. ${ }^{56}$ IL- 6 combined with IL-23 promotes Th17 responses that enhance epithelial expression of antimicrobial peptides ${ }^{57}$ and Th17 cells direct neutrophil recruitment into sites of bacterial and fungal infection. ${ }^{58}$ However, overwhelming expression of acute phase cytokines produces excessive neutrophil recruitment, resulting in tissue destruction and inflammatory tissue damage. ${ }^{56}$ Moreover, elevated Th17 responses are associated with the pathogenesis of several autoimmune and inflammatory diseases, including Crohn's disease. ${ }^{59}$ cHMOSs curbed excessive expression of Th17-polarizing cytokines including TNF- $\beta$, transforming growth factor- $\beta 1$, IL-4, and IL-17 (Figure 4). cHMOSs also attenuated expression of acute phase inflammatory cytokines, including IL-6 and IL-1 $\beta$. IL-6 controls regulatory T cell/Th17 balance ${ }^{60}$ and negatively regulates Foxp $3^{+}$regulatory $\mathrm{T}$ cell differentiation. Foxp $3^{+}$regulatory $\mathrm{T}$ cells attenuate experimental $\mathrm{NEC}^{61}$ and are present in lower amounts in NEC patients than in normal control groups. ${ }^{62}$ Decreased IL-6 promotes Foxp $3^{+}$regulatory $\mathrm{T}$ cell differentiation. Therefore, the suppression of IL-6 levels by cHMOSs may contribute to the lower incidence of NEC in infants fed human milk. ${ }^{11,17}$ Indeed, the ability of cHMOSs to accelerate maturation of immature intestinal mucosa, while modulating acute phase signaling and promoting homeostasis, may contribute to the ability of human milk feeding to decrease risk of many clinical conditions in neonates.
This modulation of basal expression of signaling agents by cHMOSs addresses the ability of cHMOSs to promote homeostasis of intrinsic cytokine levels in the absence of external influences. However, a critical feature of the immature gut is the excessive perturbation of its innate immune system by bacterial ligands. PAMPs are powerful ligands to the sentinel molecules of the innate immune system, especially the TLRs. cHMOSs modulate the response of the immature mucosa to PAMPs common in newly colonizing organisms, represented by FLA and PIC, and to a common mediator of inflammation, IL-1 $\beta$. FLA, a principal component of bacterial flagella, is a virulence factor recognized by the innate immune system via TLR5. $^{63}$ PIC, a synthetic double-stranded RNA molecule, mimics RNA virus infection $^{64}$ via stimulation of TLR3. IL-1 $\beta$ initiates and amplifies a wide variety of effects associated with innate immunity and host responses to microbial invasion and tissue injury ${ }^{65}$ mediated through stimulation of the IL-1 $\beta$ receptor. cHMOSs modulated the increased expression of a wide spectrum of cytokines in immature intestinal tissue that typically follows exposure to PAMPs and IL-1 $\beta$ (Figure 5). Two general trends were apparent: the first was that acute phase inflammatory cytokines, including IL-6, IL-8, TNF- $\alpha$, TNF- $\beta$ and MCP-1, were suppressed. The second was that cytokines involved in tissue repair and tissue homeostasis such as MIP-1- $\delta$, MIP-1- $\beta$, tissue inhibitor of matalloproteinase-2, and platelet derived growth factor were enhanced. These results strongly suggest potential anti-inflammatory and mucosal protective functions of cHMOSs in PAMP or IL-1 $\beta$-stimulated immature intestine. This is consistent with, and may account for, the decreased incidence of infant inflammatory diseases among breast-fed infants, including NEC, asthma, and inflammatory bowel disease. ${ }^{35,66}$ The direct immunomodulation described herein could be synergistic with the prebiotic effect whereby HMOSs regulate the microbiome, ${ }^{67,68}$ and the microbiome promotes recovery from chemically induced (dextran sulfate sodium) injury of the intestinal mucosa. ${ }^{69,70}$

The recognized biological functions of natural mixtures of HMOSs include inhibition of adhesion of pathogens to the cell surface receptors of their target cells, an essential first step in pathogenesis. In addition, HMOSs stimulate colonization of the infant gut with symbiotic mutualist bacteria of the microbiota (a prebiotic effect), which has several potential health benefits. $^{71}$ Both of these effects would indirectly influence inflammatory signaling in the immature intestinal mucosa. For example, dietary prebiotic oligosaccharides of plant origin are structurally dissimilar to HMOSs, but their prebiotic effect is reported to enhance Th1-dependent vaccination responses in mice through modulation of the microbiome. ${ }^{72}$ This report supports the concept that HMOSs also directly modulate the immune system of the intestinal mucosa, ${ }^{15,16}$ and provide potential pathways for their mechanism of immunomodulation.

HMOS expression is especially heterogenous, with maternal glycosyltransferase polymorphisms, notably of the fucosyltransferases, being a major factor driving variation among individuals and populations. ${ }^{73}$ Moreover, the expression of 
HMOSs is distinct in both quality and quantity between colostrum, a mother's first milk (here, before day 8 of lactation), and the mature milk that is produced from the second week of lactation onward. Variation in individual patterns of HMOS expression underlies differences in biological activities among different preparations of HMOSs. Of the eight HMOS preparations tested herein, six were from mature milks pooled from multiple mothers (pooling decreases differences because of individual genetic variation), and two were prepared from pooled colostrums. The activities described above were robust and reproducible only in the colostrum preparations. cHMOS and mHMOS compositions were compared by LC-MS. Consistent with previous reports, cHMOS preparations had higher relative amounts of complex structures, such as lacto- $\mathrm{N}$ neohexaose and monofucosyl-lacto- $N$-hexaose I. cHMOSs also included $6^{\prime}$-galactosyllactose and $3^{\prime}$-galactosyllactose, oligosaccharides not typically reported in human milk, but that had been reported in an analysis of colostrum. ${ }^{20}$ In the H4 immature human intestinal epithelial cells, these three galactosyloligosaccharides do not have the ability exhibited by cHMOS preparations to attenuate inflammation induced by FLA and IL-1 $\beta$ (Figure $6 \mathrm{c}-\mathbf{e}$ ). In contrast, $200 \mu \mathrm{g} \mathrm{ml}^{-1} 3^{\prime}$-, $6^{\prime}$-, and 4-galactosyllactoses each inhibited the inflammatory activity in $2 \mu \mathrm{g} \mathrm{ml}^{-1}$ PIC-stimulated H4 cells (Figure 6). In the presence of $200 \mathrm{ng} \mathrm{ml}^{-1}$ PIC in $\mathrm{H} 4$ cells, the induction of IL-8 protein levels was inhibited in a dose-dependent manner by $6^{\prime}$-galactosyllactose and 4-galactosyllactose from 75 to $200 \mu \mathrm{g} \mathrm{ml}^{-1}$. $3^{\prime}$-Galactosyllactose inhibited induction of IL-8 across this same range. These are within the range of concentrations measured in the cHMOS preparations: $3^{\prime}$-galactosyllactose was 47 and $79 \mu \mathrm{g} \mathrm{ml}^{-1}$, 4-galactosyllactose was 0 and $80 \mu \mathrm{g} \mathrm{ml}^{-1}$, and $6^{\prime}$-galactosyllactose was 125 and $157 \mu \mathrm{g} \mathrm{ml}^{-1}$. Thus, the galactosyllactoses may account for one of the pathways attenuated by cHMOSs.

Further research can explore additional pathways modulated by the remaining individual oligosaccharides of cHMOSs preparation. The individual roles of each cell type of the intestinal mucosa could be defined, as well as the signaling interrelationships among the different intestinal mucosal cell types. In such studies, the numbers of observations in each treatment group could be expanded to allow more fine-grained analysis of the interactions among signals than was possible in this study.

To summarize, in quiescent non-inflamed immature human intestine, cHMOSs enhance expression of genes involved in immune cell trafficking and proliferation through an ERK1/2 pathway. Simultaneously, cHMOSs heighten signaling for the recruitment of immune cells to the mucosal surface and intestinal immune development: cHMOSs stimulate expression of Th1 polarization cytokines and suppresses Th2/Th17polarizing cytokines, thereby moving Th1/Th2 balance toward that of mature tissues (Figure $7 \mathbf{b}$ ). In inflamed mucosa, cHMOSs suppress PAMP-induced release of acute phase inflammatory factors. Concurrently, cHMOSs stimulate expression of tissue repair and tissue homeostasis cytokines. Milk oligosaccharides from colostrum are more active than oligosaccharides from mature milk, and their activities are complementary and synergistic. The immature intestinal mucosa is highly inflammatory, and therefore, the greatest need for modulation of signaling coincides with the period of colostrum production. HMOSs may constitute a major innate immune system that protects the infant immature intestinal mucosa, directs maturation of the immune system, and colonizes the intestine. That cHMOSs per se are immune regulators could explain the clinical association between human milk consumption and reduced risk of early inflammatory bowel diseases. These data support use of human milk as a standard of care in the neonatal intensive care unit; moreover, individual cHMOSs may become novel prophylactic and therapeutic agents.

\section{METHODS}

Ethics. Human milk collected within 7 days after delivery (colostrum) was obtained from the Mothers' Milk Bank Northeast MMBNE (Newton, MA). Human small intestine samples were a gift of Dr Nanda Nanthakumar (Mucosal Immunology Lab, Massachusetts General Hospital, Harvard Medical School, Boston, MA) approved by the Committee for the Protection of Human Subjects from Research Risks (Brigham and Women's Hospital, Boston, MA), and the Human Investigation Committee (Massachusetts General Hospital). The research was approved by Partners Human Research Committee.

Purification of HMOSs. HMOSs were prepared ${ }^{74}$ as cHMOSs or mHMOSs by pooling individual samples into 1-liter batches representing 20-50 donors. During production of early milk, volumes are limited. Even by defining any early milk ( $0-7$ days) as 'colostrum', only two batches of colostrum could be prepared from all of the milks of convenience available to us. In contrast, mature milk was not limiting and six batches were prepared to increase the statistical power of the comparison. After centrifugation at $3,000 \times g$ for $40 \mathrm{~min}$ at $4{ }^{\circ} \mathrm{C}$, decanting eliminated the cream. The clear aqueous phase was made $67 \%$ ethanol at $4{ }^{\circ} \mathrm{C}$ and stirred overnight to precipitate protein. After centrifugation at $3,000 \times g$ for $40 \mathrm{~min}$ at $4{ }^{\circ} \mathrm{C}$, and decanting through a filter, the crude oligosaccharide fraction was applied to a 2-1 charcoal celite column, the column was washed with $5 \%$ ethanol, and the HMOSs were eluted with $65 \%$ ethanol and dried by rotary evaporation followed by lyophilization. Potential endotoxin contamination was removed by passing the HMOS preparation through a polymyxin $\mathrm{B}$ column until the content of endotoxin activity was below 0.01 units (Limulus Amebocyte Lysate, LAL, GenScript, Piscataway, NJ). HMOSs from pooled mHMOSs was prepared identically. HMOS preparations were lyophilized and stored at $-20^{\circ} \mathrm{C}$ until use. The two cHMOS and six mHMOS preparations were compared for content of individual oligosaccharides by LC-MS, and their activities compared in epithelial cells by IL-8 ELISA.

Fetal intestinal organ culture. Cultures of fetal small intestine samples ${ }^{24}$ utilized fresh tissue samples from elective prostaglandin/ saline-induced therapeutic abortion of 22-week fetuses that had been transported to the laboratory in ice-cold fresh Dulbecco's modified Eagle's medium/F12 medium containing $40 \mathrm{mg} \mathrm{ml}^{-1}$ of penicillin and gentamicin. Twenty-two weeks is approximately the age of extremely low birthweight neonates but, that notwithstanding, the intestine is already differentiated into colon, ileum, jejunum, and duodenum. Each experimental group is the aggregate of duplicate independent samples from three individuals. Tissues were rinsed in fresh ice-cold Dulbecco's modified Eagle's medium/F12 media and the mesentery and connective tissues were removed. The intestine was opened longitudinally with scissors and the mucosal side was gently washed with medium to remove meconium and other debris. The tissue was oriented under the dissecting microscope with the mucosal side up, cut into cubes of 
$0.5 \mathrm{~cm}$ on edge, transferred to 24 -well cell culture plates, and incubated with $0.5 \mathrm{ml}$ of Dulbecco's modified Eagle's medium/F12 media at 5\% $\mathrm{CO}_{2}$ and $37^{\circ} \mathrm{C}$.

Treatment of intestinal mucosa with PAMP-related stimuli and cHMOSs. For each intestine, the inflammatory state of the cultured intestinal cubes was determined as IL-8 content of the supernatant. The tissue was deemed useful for these experiments only if the inflammatory state for most wells was at quiescent baseline levels $\left(\sim 100 \mathrm{pg} \mathrm{ml}^{-1}\right)$, indicating that the gut was not already inflamed due to prior damage. Half of the healthy samples were treated with $20 \mu \mathrm{l}$ HMOSs in PBS to achieve a final physiological concentration of $5 \mathrm{mg} \mathrm{ml}^{-1}$ in each well, and further incubated (basal, cHMOSs) for 2 days; the other half received $20 \mu \mathrm{l}$ of PBS alone (basal). After a 2-day incubation, some of the basal and basal-HMOS groups were further supplemented with $20 \mu \mathrm{l}$ of an individual PAMP or IL-1 $\beta$ in PBS to achieve a final concentration per well of $10 \mathrm{ng} \mathrm{ml}^{-1} \mathrm{IL}-1 \beta, 100 \mathrm{ng} \mathrm{ml}^{-1}$ FLA, or $10 \mathrm{ng} \mathrm{ml}^{-1} \mathrm{PIC}^{24} \mathrm{PBS}$ alone was used for the unstimulated controls. Tissues were maintained in $5 \% \mathrm{CO}_{2}$ at $37^{\circ} \mathrm{C}$ for an additional $18 \mathrm{~h}$. Supernatants were collected for IL-8 ELISA analysis. Tissues were homogenized on ice in lysis buffer (Cell Signaling, Danvers, MA) supplemented with protease inhibitor cocktail (Cell Signaling); protein concentration was determined by BCA Protein Assay (Pierce, Rockford, IL).

Treatment of immature intestinal epithelial H4 cells with PAMPrelated stimuli and cHMOSs. Aliquots of the immature intestinal epithelial cell line H4 ( $10^{3}$ cells) were cultured for 2 days in 96-well plates, whereupon they received $4 \mu \mathrm{l}$ of milk oligosaccharides (final concentrations: HMOSs, $5 \mathrm{mg} \mathrm{ml}^{-1} ; 3^{\prime}-$, 4-, and $6^{\prime}$-galactosyllactoses [Carbosynth, Compton, Berkshire, UK], $200 \mu \mathrm{g} \mathrm{ml}^{-1}$ ), or PBS alone (basal). After an additional 2 days of incubation, designated wells received $20 \mu \mathrm{l}$ of individual PAMPs or IL- $1 \beta$ in PBS to achieve a final concentration of $1 \mu \mathrm{g} \mathrm{ml}^{-1}$ of IL-1 $\beta 200 \mathrm{ng} \mathrm{ml}^{-1}$ of FLA or $2 \mu \mathrm{g} \mathrm{ml}^{-1}$ of PIC. These concentrations had been found to be effective in a variety of intestinal epithelial cell lines in previous work (unpublished data). The unstimulated control received $20 \mu \mathrm{l}$ of PBS without PAMP-related molecules. Cells were maintained in DMEM containing $10 \%$ fetal bovine serum and $30 \mathrm{ng} \mathrm{ml}^{-1}$ epithelial growth factor in $5 \% \mathrm{CO}_{2}$ at $37^{\circ} \mathrm{C}$ for $18 \mathrm{~h}$. Supernatants were collected for IL-8 ELISA analysis. Results were normalized to cell number.

IL-8 ELISA. IL-8 was measured in supernatant after brief centrifugation according to the manufacturer's instructions (R\&D Systems, Minneapolis, MN). The color generated at $450 \mathrm{~nm}$ was measured on a Versa Max plate reader (Molecular Devices, Sunnyvale, CA). Values were normalized to the total protein concentrations of tissue lysates.

Tissue RNA extraction. RNA was extracted from homogenized human immature intestinal tissue using Trizol (Invitrogen, Grand Island, NY). Purification was by RNeasy minikit and Qiashredder columns from Qiagen (Valencia, CA) according to the manufacturer's instructions.

$\mathbf{R T}^{\mathbf{2}}$ profiler PCR array. Transcription of human inflammatory cytokines and receptor genes was measured in a human $\mathrm{RT}^{2}$ Profiler PCR Inflammatory Cytokines and Receptors Array (SABiosciences, PAHS-011ZA, Qiagen). Reverse transcription of $2 \mu \mathrm{g}$ RNA from tissue was by $\mathrm{RT}^{2}$ First Strand Kit, and amplified samples were applied to PCR array plates. The $\mathrm{RT}^{2}$ Profiler arrays were probed with SYBR green/fluorescein master mix using the Profiler PCR array system. A two-step real-time PCR reaction was initiated at $95^{\circ} \mathrm{C}(10 \mathrm{~min})$ for 1 cycle and followed alternation of $95^{\circ} \mathrm{C}(15 \mathrm{~s})$ and $60^{\circ} \mathrm{C}(1 \mathrm{~min})$ for 40 cycles (7500 Fast Real-Time PCR System, Applied Biosystems, Grand Island, NY). Data were analyzed using automated baseline and threshold cycle detection and interpreted via an excel-based PCR array data analytical tool from SABiosciences. Results were normalized to the expression of housekeeping genes. Upregulation $>3.5$-fold or downregulation to below 0.8 demarked significant differences.
Antibody array of human signaling cytokines. Changes in cytokine and chemokine expression in intestinal tissue lysates were monitored by the RayBio Human Inflammation Antibody Array III (RayBiotech, Norcross, GA). Homogenized tissue lysates containing $40 \mu \mathrm{g}$ of protein were diluted in blocking buffer and bound to array membranes overnight at $4{ }^{\circ} \mathrm{C}$. Membranes were processed according to the manufacturer's instructions and relative quantities of inflammatory cytokines were visualized by X-ray film exposure. Spots were quantified using Image J analysis software (Eastman Kodak, Rochester, NY). Upregulation of $>1.2$-fold and downregulation of $<0.8$-fold were deemed significant.

Ingenuity pathways analysis. Data from the $\mathrm{RT}^{2}$ profiler $\mathrm{PCR}$ array were imported into Ingenuity Systems (www.ingenuity.com) to perform pathway analysis. Both up- and downregulated identifiers were defined as value parameters. The generated networks were rank ordered according to their significance score. Significance of biofunctional and canonical pathways are indicated by the Fisher's exact test $P$-value. Canonical pathways were grouped into signaling pathway networks. Canonical pathways were rank ordered according to the IPA-generated relevance ratios. This pathway analysis also generated networks of differentially regulated genes based on previously known associations between genes or proteins, but independent of established canonical pathways.

HMOS profile by LC-MS. Purified HMOSs (1 mg) were dissolved in $1 \mathrm{ml}$ of Milli-Q water and reduced with an excess of $\mathrm{NaBH}_{4}$ at $25^{\circ} \mathrm{C}$ for $16 \mathrm{~h}$. The reaction was stopped by dropwise addition of glacial HAc and samples were evaporated under nitrogen. After two washings with $\mathrm{MeOH} / \mathrm{AcOH} 95 / 5$ and an additional two with $\mathrm{MeOH}$, samples were dissolved in $2 \mathrm{ml}$ distilled water and desalted with $25 \mu \mathrm{l}$ of DOWEX$50 \mathrm{~W}$ resin (Sigma, St Louis, MO), which had been prewashed with $1 \mathrm{M}$ $\mathrm{HCl}$. Each fraction $(3 \mu \mathrm{l}$ ) was analyzed by LC-MS (Agilent 1100 HPLC equipped with an Agilent ESI-TOF 6220 mass spectrometer, Agilent Technologies, Santa Clara, CA). LC separation was achieved on a graphitized carbon stationary phase (Hypercarb, $100 \times 2.1 \mathrm{~mm}$, Thermo Fisher Scientific, Waltham, MA). The mobile phase was a gradient of AcCN (B) in $\mathrm{H}_{2} \mathrm{O}(\mathrm{A})$ at a flow rate of $0.20 \mathrm{ml} \mathrm{min}^{-1}$ as follows: $0.0-2.0 \mathrm{~min}, 0 \% \mathrm{~B}$; $2.0-6.0 \mathrm{~min}, 0-5 \% \mathrm{~B}$; $6.0-9 \mathrm{~min}, 5 \% \mathrm{~B}$; 9.0-20.0 min, 5-12\% B; 20.0-22.0 min, $12 \%$ B; 22.0-26.0 min, $12-20 \%$ B; 26.0-32.0 min, 20-50\% B; 32.0-35.0 min, 50-90\% B; 35.0-40.0 min, $90 \% \mathrm{~B} ; 40.0-41.0 \mathrm{~min}, 90-0 \% \mathrm{~B}$. Each injection was followed by 10 -min equilibration at $0 \% \mathrm{~B}$. Ion spray voltage was $-3,500 \mathrm{~V}$ and the gas temperature was $350^{\circ} \mathrm{C}$. The fragmentor was set at $70 \mathrm{~V}$. Data acquisition were performed in the negative mode starting at minute 6 of the elution, covering the $\mathrm{m} / \mathrm{z}$ range $350-2300$ at a 1.03 spectrum $^{-1}$ scan rate.

Statistical analysis. All data represent duplicate independent values from each tissue from at least three different tissues $(n=6)$ and are presented as the mean \pm s.e.m. The significance of differences between mean values was determined using two-way repeated-measures analysis of variance, which incorporates posttest Bonferroni correction for multiple comparisons in Prism software (Graph Pad Software, San Diego, CA). Comparison of differences between cHMOS and mHMOS activities was evaluated by Fisher's exact ratios. Differences with $P$-values equal to or lower than 0.05 were considered significant.

SUPPLEMENTARY MATERIAL is linked to the online version of the paper at http://www.nature.com/mi

\section{ACKNOWLEDGMENTS}

This work was partially supported by the NIH grants (R01HD059140, U01Al075563, and P01HD013021) and Abbott Nutrition. We thank Dr Nanda Nanthakumar (Massachusetts General Hospital, Harvard Medical School) for providing human intestinal tissue and assisting with organ culture, and Dr Lei Lu (Massachusetts General Hospital, Harvard Medical School) for providing $\mathrm{H} 4$ cells. 


\section{DISCLOSURE}

DSN owns stock in Glycosyn, LLC, which makes HMOSs. This potential conflict is being managed by Boston College.

(c) 2014 Society for Mucosal Immunology

\section{REFERENCES}

1. Mannick, E. \& Udall, J.N. Jr. Neonatal gastrointestinal mucosal immunity. Clin Perinatol 23, 287-304 (1996).

2. Bailey, M. et al. The development of the mucosal immune system pre- and post-weaning: balancing regulatory and effector function. Proc Nutr Soc 64, 451-457 (2005).

3. Rognum, T.O., Thrane, S., Stoltenberg, L., Vege, A. \& Brandtzaeg, P. Development of intestinal mucosal immunity in fetal life and the first postnatal months. Pediatr Res 32, 145-149 (1992).

4. Schnabl, K.L., Van Aerde, J.E., Thomson, A.B. \& Clandinin, M.T. Necrotizing enterocolitis: a multifactorial disease with no cure. World $J$ Gastroenterol 14, 2142-2161 (2008).

5. Lin, P.W. \& Stoll, B.J. Necrotising enterocolitis. Lancet 368, 1271-1283 (2006).

6. Schirbel, A. \& Fiocchi, C. Targeting the innate immune system in pediatric inflammatory bowel disease. Exp Rev Gastroenterol Hepatol 5, 33-41 (2011).

7. Buescher, E.S. Anti-inflammatory characteristics of human milk: how, where, why. Adv Exp Med Biol 501, 207-222 (2001)

8. Ballard, O. \& Morrow, A.L. Human milk composition: nutrients and bioactive factors. Pediatr Clin North Am 60, 49-74 (2013).

9. Kelly, D. \& Coutts, A.G. Early nutrition and the development of immune function in the neonate. Proc Nutr Soc 59, 177-185 (2000).

10. Schanler, R.J., Shulman, R.J. \& Lau, C. Feeding strategies for premature infants: beneficial outcomes of feeding fortified human milk versus preterm formula. Pediatrics 103 (6 Pt 1), 1150-1157 (1999).

11. Lucas, A. \& Cole, T.J. Breast milk and neonatal necrotising enterocolitis. Lancet 336, 1519-1523 (1990).

12. Klement, E., Cohen, R.V., Boxman, J., Joseph, A. \& Reif, S. Breastfeeding and risk of inflammatory bowel disease: a systematic review with metaanalysis. Am J Clin Nutr 80, 1342-1352 (2004).

13. Newburg, D.S., Ruiz-Palacios, G.M. \& Morrow, A.L. Human milk glycans protect infants against enteric pathogens. Annu Rev Nutr 25, 37-58 (2005).

14. Kunz, C., Rudloff, S., Baier, W., Klein, N. \& Strobel, S. Oligosaccharides in human milk: structural, functional, and metabolic aspects. Annu Rev Nutr 20, 699-722 (2000).

15. Kunz, C. \& Rudloff, S. Potential anti-inflammatory and anti-infectious effects of human milk oligosaccharides. Adv Exp Med Biol 606, 455-465 (2008).

16. Naarding, M.A. et al. Lewis $X$ component in human milk binds. J Clin Invest 115, 3256-3264 (2005).

17. Jantscher-Krenn, E. et al. The human milk oligosaccharide disialyllacto-Ntetraose prevents necrotising enterocolitis in neonatal rats. Gut 61 , 1417-1425 (2012)

18. Coppa, G.V., Pierani, P., Zampini, L., Carloni, I., Carlucci, A. \& Gabrielli, O. Oligosaccharides in human milk during different phases of lactation. Acta Paediatr Supp/ 88, 89-94 (1999).

19. Chaturvedi, P. et al. Fucosylated human milk oligosaccharides vary between individuals and over the course of lactation. Glycobiology 11, 365-372 (2001).

20. Sumiyoshi, W. et al. Galactosyllactoses in the milk of Japanese women: changes in concentration during the course of lactation. J Appl Glycosci 51, 341-344 (2004).

21. Zarember, K.A. \& Godowski, P.J. Tissue expression of human Toll-like receptors and differential regulation of Toll-like receptor mRNAs in leukocytes in response to microbes, their products, and cytokines. J Immunol 168, 554-561 (2002).

22. Humke, E.W., Shriver, S.K., Starovasnik, M.A., Fairbrother, W.J. \& Dixit, V.M. ICEBERG: a novel inhibitor of interleukin-1beta generation. Cell 103 99-111 (2000).

23. Vogel, C. \& Marcotte, E.M. Insights into the regulation of protein abundance from proteomic and transcriptomic analyses. Nat Rev Genet 13, 227-232 (2012).
24. Nanthakumar, N.N., Fusunyan, R.D., Sanderson, I. \& Walker, W.A. Inflammation in the developing human intestine: a possible pathophysiologic contribution to necrotizing enterocolitis. Proc Natl Acad Sci USA 97, 6043-6048 (2000)

25. Youn, B.S. et al. Molecular cloning of leukotactin-1: a novel human betachemokine, a chemoattractant for neutrophils, monocytes, and lymphocytes, and a potent agonist at CC chemokine receptors 1 and 3 . J Immunol 159, 5201-5205 (1997).

26. Yang, L., Froio, R.M., Sciuto, T.E., Dvorak, A.M., Alon, R. \& Luscinskas, F.W. ICAM-1 regulates neutrophil adhesion and transcellular migration of TNF-alpha-activated vascular endothelium under flow. Blood 106, 584-592 (2005).

27. Clements, J.M. et al. Two PDGF-B chain residues, arginine 27 and isoleucine 30, mediate receptor binding and activation. EMBO J 10, 4113-4120 (1991)

28. Morgunova, E., Tuuttila, A., Bergmann, U. \& Tryggvason, K. Structural insight into the complex formation of latent matrix metalloproteinase 2 with tissue inhibitor of metalloproteinase 2. Proc Natl Acad Sci USA 99, 7414-7419 (2002)

29. Baggiolini, M., Walz, A. \& Kunkel, S.L. Neutrophil-activating peptide-1/ interleukin 8, a novel cytokine that activates neutrophils. J Clin Invest 84, 1045-1049 (1989).

30. McCracken, V.J. \& Lorenz, R.G. The gastrointestinal ecosystem: a precarious alliance among epithelium, immunity and microbiota. Cell Microbio/ 3, 1-11 (2001).

31. Nanthakumar, N. et al. The Mechanism of excessive intestinal inflammation in necrotizing enterocolitis: an immature innate immune response. PLoS One 6, e17776 (2011).

32. Kuo, S., El Guindy, A., Panwala, C.M., Hagan, P.M. \& Camerini, V. Differential appearance of T cell subsets in the large and small intestine of neonatal mice. Pediatr Res 49, 543-551 (2001).

33. Levy, O. Innate immunity of the newborn: basic mechanisms and clinical correlates. Nat Rev Immunol 7, 379-390 (2007).

34. Abrahamsson, T.R., Sandberg Abelius, M., Forsberg, A., Bjorksten, B. \& Jenmalm, M.C.A. Th1/Th2-associated chemokine imbalance during infancy in children developing eczema, wheeze and sensitization. Clin Exp Allergy 41, 1729-1739 (2011).

35. Gartner, L.M. et al. Breastfeeding and the use of human milk. Pediatrics 115, 496-506 (2005).

36. Newburg, D.S. Neonatal protection by an innate immune system of human milk consisting of oligosaccharides and glycans. J Anim Sci 87 (Suppl 13), 26-34 (2009).

37. Goldman, A.S., Goldblum, R.M. \& Hanson, L.A. Anti-inflammatory systems in human milk. Adv Exp Med Biol 262, 69-76 (1990).

38. Eiwegger, T. et al. Human milk-derived oligosaccharides and plantderived oligosaccharides stimulate cytokine production of cord blood T-cells in vitro. Pediatr Res 56, 536-540 (2004).

39. Armogida, S.A., Yannaras, N.M., Melton, A.L. \& Srivastava, M.D. Identification and quantification of innate immune system mediators in human breast milk. Allergy Asthma Proc 25, 297-304 (2004).

40. Kramer, M.S. et al. Promotion of breastfeeding intervention trial (PROBIT): a randomized trial in the Republic of Belarus. JAMA 285, 413-420 (2001).

41. Hofmann, S.R. et al. Cytokines and their role in lymphoid development, differentiation and homeostasis. Curr Opin Allergy Clin Immunol 2, 495-506 (2002).

42. Takeuchi, H., Fujimoto, A., Tanaka, M., Yamano, T., Hsueh, E. \& Hoon, D.S. CCL21 chemokine regulates chemokine receptor CCR7 bearing malignant melanoma cells. Clin Cancer Res 10, 2351-2358 (2004).

43. Sallusto, F., Mackay, C.R. \& Lanzavecchia, A. Selective expression of the eotaxin receptor CCR3 by human T helper 2 cells. Science 277, 2005-2007 (1997).

44. Garcia-Zepeda, E.A. et al. Human monocyte chemoattractant protein (MCP)-4 is a novel CC chemokine with activities on monocytes, eosinophils, and basophils induced in allergic and nonallergic inflammation that signals through the CC chemokine receptors (CCR)-2 and -3 . $\mathrm{J}$ Immunol 157, 5613-5626 (1996).

45. Ahuja, S.K. \& Murphy, P.M. The CXC chemokines growth-regulated oncogene (GRO) alpha, GRObeta, GROgamma, neutrophil-activating peptide-2, and epithelial cell-derived neutrophil-activating peptide-78 are potent agonists for the type B, but not the type A, human interleukin-8 receptor. J Biol Chem 271, 20545-20550 (1996). 
46. Shannon, L.A., Calloway, P.A., Welch, T.P. \& Vines, C.M. CCR7/CCL21 migration on fibronectin is mediated by phospholipase $\mathrm{C} \gamma 1$ and ERK $1 / 2$ in primary T lymphocytes. J Biol Chem 285, 38781-38787 (2010).

47. Meloche, S. \& Pouyssegur, J. The ERK1/2 mitogen-activated protein kinase pathway as a master regulator of the G1- to S-phase transition. Oncogene 26, 3227-3239 (2007).

48. Maheshwari, A. \& Zemlin, M. Ontogeny of the intestinal immune system. Immunol Infect 2, 18-26 (2006).

49. Neurath, M.F., Finotto, S. \& Glimcher, L.H. The role of Th1/Th2 polarization in mucosal immunity. Nat Med 8, 567-573 (2002).

50. Kidd, P. Th1/Th2 balance: the hypothesis, its limitations, and implications for health and disease. Altern Med Rev 8, 223-246 (2003).

51. Randolph, D.A., Huang, G., Carruthers, C.J., Bromley, L.E. \& Chaplin, D.D. The role of CCR7 in TH1 and TH2 cell localization and delivery of B cell help in vivo. Science 286, 2159-2162 (1999).

52. Vigne, S. et al. IL-36 signaling amplifies Th1 responses by enhancing proliferation and Th1 polarization of naive CD4 + Tcells. Blood 120, 3478-3487 (2012).

53. Shaffer, A.L., Yu, X., He, Y., Boldrick, J., Chan, E.P. \& Staudt, L.M. BCL-6 represses genes that function in lymphocyte differentiation, inflammation, and cell cycle control. Immunity 13, 199-212 (2000).

54. Takatsu, K. Interleukin 5 and B cell differentiation. Cytokine Growth Factor Rev 9, 25-35 (1998).

55. Clinton, C. Development of the infant immune function and the effects of breast milk. Natural Med J 2, 3-6 (2010).

56. Kumar, V. \& Sharma, A. Neutrophils: Cinderella of innate immune system. Int Immunopharmacol 10, 1325-1334 (2010).

57. Liang, S.C. et al. Interleukin (IL)-22 and IL-17 are coexpressed by Th17 cells and cooperatively enhance expression of antimicrobial peptides. $J$ Exp Med 203, 2271-2279 (2006).

58. Korn, T., Bettelli, E., Oukka, M. \& Kuchroo, V.K. IL-17 and Th17 Cells. Annu Rev Immunol 27, 485-517 (2009).

59. Maddur, M.S., Miossec, P., Kaveri, S.V. \& Bayry, J. Th17 cells: biology, pathogenesis of autoimmune and inflammatory diseases, and therapeutic strategies. Am J Pathol 181, 8-18 (2012).

60. Kimura, A. \& Kishimoto, T. IL-6: regulator of Treg/Th17 balance. Eur J Immunol 40, 1830-1835 (2010).

61. Dingle, B.M., Liu, Y., Fatheree, N.Y., Min, J., Rhoads, J.M. \& Tran, D.Q. FoxP3(+) Regulatory T cells attenuate experimental necrotizing enterocolitis. PLoS One 8, e82963 (2013).
62. Weitkamp, J.-H. et al. Necrotising enterocolitis is characterised by disrupted immune regulation and diminished mucosal regulatory (FOXP3)/effector (CD4, CD8) T cell ratios. Gut 62, 73-82 (2012).

63. Hayashi, F. et al. The innate immune response to bacterial flagellin is mediated by Toll-like receptor 5. Nature 410, 1099-1103 (2001).

64. De Miranda, J., Yaddanapudi, K., Hornig, M. \& Lipkin, W.I. Astrocytes recognize intracellular polyinosinic-polycytidylic acid via MDA-5. FASEB J 23, 1064-1071 (2009).

65. Eder, C. Mechanisms of interleukin-1 $\beta$ release. Immunobiology 214, 543-553 (2009).

66. Sullivan, S. et al. An exclusively human milk-based diet is associated with a lower rate of necrotizing enterocolitis than a diet of human milk and bovine milk-based products. J Pediatr 156, 562-567, e561 (2010).

67. Yu, Z.T. et al. The principal fucosylated oligosaccharides of human milk exhibit prebiotic properties on cultured infant microbiota. Glycobiology 23 , 169-177 (2013).

68. Yu, Z.T., Chen, C. \& Newburg, D.S. Utilization of major fucosylated and sialylated human milk oligosaccharides by isolated human gut microbes. Glycobiology 23, 1281-1292 (2013).

69. Nanthakumar, N.N., Meng, D. \& Newburg, D.S. Glucocorticoids and microbiota regulate ontogeny of intestinal fucosyltransferase 2 requisite for gut homeostasis. Glycobiology 23, 1131-1141 (2013).

70. Weiss, G.A. \& Hennet, T. The role of milk sialyllactose in intestinal bacterial colonization. Adv Nutr 3, 483S-488S (2012).

71. Coppa, G.V., Bruni, S., Morelli, L., Soldi, S. \& Gabrielli, O. The first prebiotics in humans: human milk oligosaccharides. J Clin Gastroenterol 38, S80-S83 (2004).

72. Vos, A.P. et al. Dietary supplementation of neutral and acidic oligosaccharides enhances Th1-dependent vaccination responses in mice. Pediatr Allergy Immunol 18, 304-312 (2007).

73. Newburg, D.S. et al. Innate protection conferred by fucosylated oligosaccharides of human milk against diarrhea in breastfed infants. Glycobiology 14, 253-263 (2004).

74. Crane, J.K., Azar, S.S., Stam, A. \& Newburg, D.S. Oligosaccharides from human milk block binding and activity of the Escherichia coli heatstable enterotoxin (STa) in T84 intestinal cells. J Nutr 124, 2358-2364 (1994). 